\title{
Pricing, Carbon Footprint and Signaling Game
}

\author{
ZhiYong Tian ${ }^{1,}$ a , RuiPing Yuan ${ }^{2, b}$ and LingYu Huo ${ }^{3, c}$ \\ 1,2,3 School of Information, Beijing Wuzi University, Beijing, China \\ atianbox@126.com, bangelholyping@163.com, ’sboerp@163.com
}

\begin{abstract}
Keywords: Carbon Footprint, Pricing, Signaling Game, Equilibrium
Abstract. Taking carbon footprint as the signal of production quantity, it creates the signaling game model of pricing between firm and consumer. By graphic analysis tools, such as indifference curve, it researches deeply the equilibriums of the model, such separating, pooling and hybrid equilibriums. The results show that there exists the only equilibrium in the case of low tech firm(LTF) does not envy high tech firm(HTF). while LTF envying HTF, there exist separating, pooling and hybrid equilibriums. But pooling and hybrid equilibriums are not robust. They cannot be realized without the aid of external forces, such as regulations and administrative powers. The separating equilibrium is self enforced, and the emission reduction at the separating point is larger than that in the case of no envy. So it is advantageous to emission reduction. The research is meaningful for firm's decision on carbon footprint and pricing especially in the market scenario that carbon label can be used as the signal of product quantity.
\end{abstract}

\section{Introduction}

Recently, low carbon research has become hot topics. There emerges a large number of literatures. These literatures can be classified into four types roughly, such as carbon footprint(CF) calculation, regulation policy, market mechanism and social awareness. In the researches of CF calculation, Christopher et al.(2008) ${ }^{[1]}$ calculates the CF of families in U.S., Paul et al.(2008) ${ }^{[2]}$ compares the ten CF calculators in U.S., Susan Cholette et al.(2009) ${ }^{[3]}$ calculates the CF of the transportation and storage of wine. There are also the researches aiming at ensure the public data of CF authority and objectivity, e.g. the practice guides and PAS 2050 released by IPCC, Carbon Trust and other international organizations. As for regulation, the literatures takes $\mathrm{CF}$ as strict constraints of decisions, e.g. Absi et al.(2016) ${ }^{[4]}$ research the order quantity problem in the constraints of carbon emissions, and other literatures such as Hua et al.(2011) ${ }^{[5]}$ and Chen et al.(2013) ${ }^{[6]}$ and etc. The literatures of market mechanism either internalize $\mathrm{CF}$ as cost or suppose there exists carbon trading market, e.g. Rui Zhao et al.(2012) ${ }^{[7]}$ considers government's rewards and punishments for carbon emissions, Li S et $\mathrm{al}(2012)^{[8]}$ is based on the suppose of carbon trading market, Bai et al.(2016) ${ }^{[9]}$ is for carbon tax and carbon cap-and-trade, and etc.. Social awareness refers the increasing of consumer awareness of low carbon, e.g. more and more consumers are becoming aware of SD issues (Wakland et al.(2009) ${ }^{[10]}$, Blengini et al.(2010) $\left.{ }^{[11]}\right)$. It is also related to the enhancement of firm's social responsibility, e.g. many progressive companies, such as Walmart, Tesco, Hewlett Packard and Patagonia, have capitalized on the opportunities of green supply chain management. All these can influence demand and supply of market, which can effect firm's decision. But there are few literatures focusing on these except for Du et. al.(2013) ${ }^{[12]}$, Hovelaque et. al.(2015) ${ }^{[13]}$ and etc.

Regulation cannot be implemented in large scale for its high cost and implementation difficulty. Social low carbon awareness has optimistic prospect, but its effect is very limited at present. Market mechanism is more operational and has fast effect relatively. So this literature focuses on it. But being different form taking the cost and revenue about low carbon into market effects, we assume a new market scenes, the reason for consumer preference for low carbon products is not the increasing of consumer low carbon awareness but taking low carbon as the basis for judging product quality. So, firm takes emission reduction as a signal to consumers about the quality of its products. The research is from the new perspective. The paper is organized as follows: Section 2 is modeling, and it describes 
the application settings and proposes the signal games model. Section 3 is the analysis of the game by indifference curve. Section 4 summaries the paper and provides further researches.

\section{Modeling}

Many firms have adopted carbon label for their products, which has become the important way for firms to reveal their endeavor and achievement in emission reduction(Liu et al.(2016 ${ }^{[14]}$ ). Certainly, carbon label should be made by the third professional organization to ensure its authority. In the market, firms can be classified into HTF and LTF according to technology. As for product quality, the product of HTF is high quality, the product of LTF is low quality. So we take the tech of firm and the quality of its product as equivalence. As for emission reduction, the costs of HTF are larger than that of LTF to the same reduction amount. For consumers are willing to pay high price for high quality product, HTF has motivation to transfer its signal. Of course, there are many ways for consumers to estimate the quality of product. But we ignore these methods here. Then, firm and consumer both take carbon label as the information to estimate the quality. There are two decisions, carbon label selection and pricing in signal transmitting. The former decision is done by firm. For pricing should take consumers' recognition and theirs preference, so pricing can be done by consumers. This process forms signaling game (Gibbons $\left.(2015)^{[15]}\right)$. The sequence of the game is as follow:

1) Nature chooses the tech levels of firm, high tech or low tech. Denote q as the probability of high tech.

2) Firm knows its tech level, and selects emissions to publish its carbon label. Denote e as emissions.

3) Consumers estimate the quality of product by the carbon label, and decide the price.

The payoff of firm is $p(e)-c(v, e)$, where $p(e)$ denotes the price with respect to emission $e$, it is the function of $\mathrm{e}$. $\mathrm{v}$ is the tech level of firm or the quality level of product. $\mathrm{c}(\mathrm{v}, \mathrm{e})$ is product costs per unit when the tech level of firm is $\mathrm{v}$ and its emission is e.

The payoff of consumers is $\mathrm{f}(\mathrm{v}, \mathrm{e})=\mathrm{u}(\mathrm{v}, \mathrm{e})-\mathrm{p}(\mathrm{e})$, where $\mathrm{u}(\mathrm{v}, \mathrm{e})$ is the utility of consumers when the tech level and emission of firm are $\mathrm{v}$ and $\mathrm{e}$. If consumers don't buy any product, the payoff is 0 . Suppose $\mathrm{u}_{v}(v, e)>0$, which means the value $\mathrm{u}$ increases with $\mathrm{v}$.

Suppose the state of market is clearing, which means the strategy of firm's emission selection is 0 and consumers not buying. We don't consider the effect of price on demand. Suppose the marginal cost for emission reduction of LTF is larger than HTF, which means $c_{e}(L, e)>c_{e}(H, e)$. It can be shown by Fig. 1 with indifference curve.

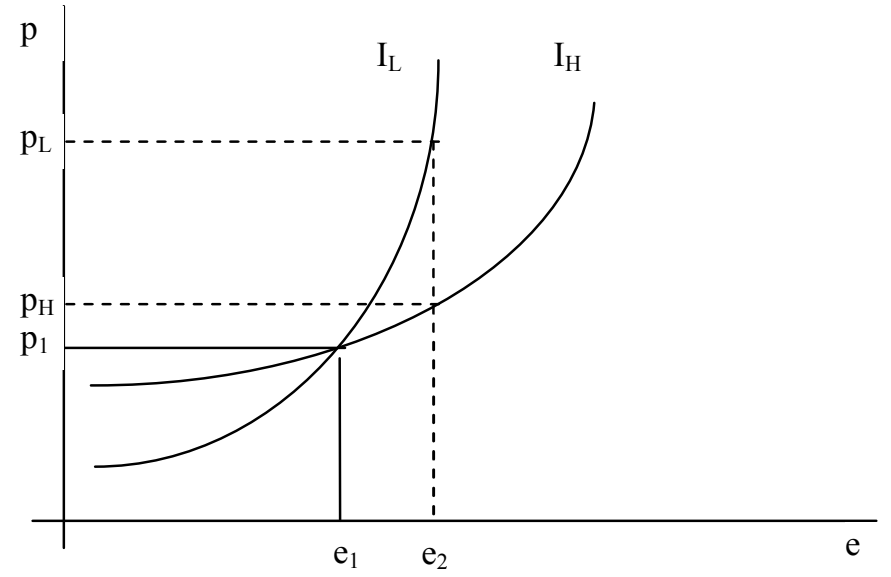

Fig. 1 The indifference curves of two types of firms 


\section{Analysis of the Game}

The game above with complete information is very simple. So, we only consider the incomplete case. In this setting, the relationship between the indifference curve of the two types of firms is very important. The relationship can be divided into envy and no envy cases., being discussed as follows.

No Envy Cases. For the difference curve of LTF is deeper than HTF, the slope and value of $f(v, e)$ for LTF is smaller and lower than HTF. The relations can be shown as Fig. 2.

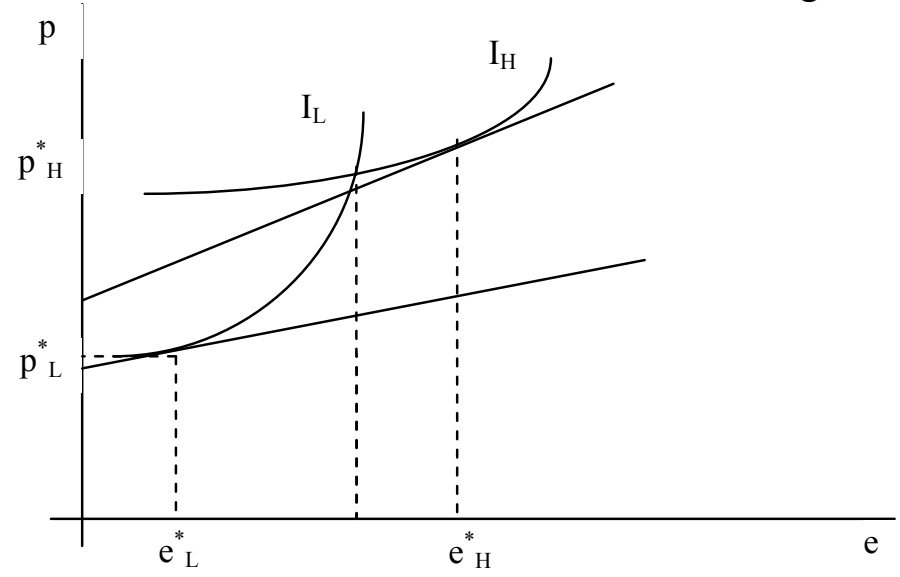

Fig. 2 The indifference curves with no envy

Due to Fig.2, for LTF there exists $p^{*}(L)-c\left(L, e_{L}^{*}\right)>p^{*}(H)-c\left(L, e_{H}^{*}\right)$. It means the dominant strategy of LTF is to tell the truth. The reason is that the costs of LTF choosing emission reduction $e_{H}^{*}$ is larger than the price that consumers pay for HTF.

In analogy with LTF, for HTF there exists $p^{*}(H)-c\left(H, e_{H}^{*}\right)>p^{*}(L)-c\left(H, e_{L}^{*}\right)$, which means the dominant strategy of HTF is to tell the truth too.

So in the case of no envy, there is the only Nash equilibrium: LTF selects low emission reduction $e_{L}^{*}$, HTF selects high emission reduction $e_{H}^{*}$, and consumers select low price $p_{L}^{*}$ with respect to $e_{L}^{*}$, and high price $p_{H}^{*}$ with respect to $e_{H}^{*}$. But this case is very rare in practice.

Envy Cases. We can only consider the case of LTF envying HTF. It can be shown as Fig. 3 with indifference curves.

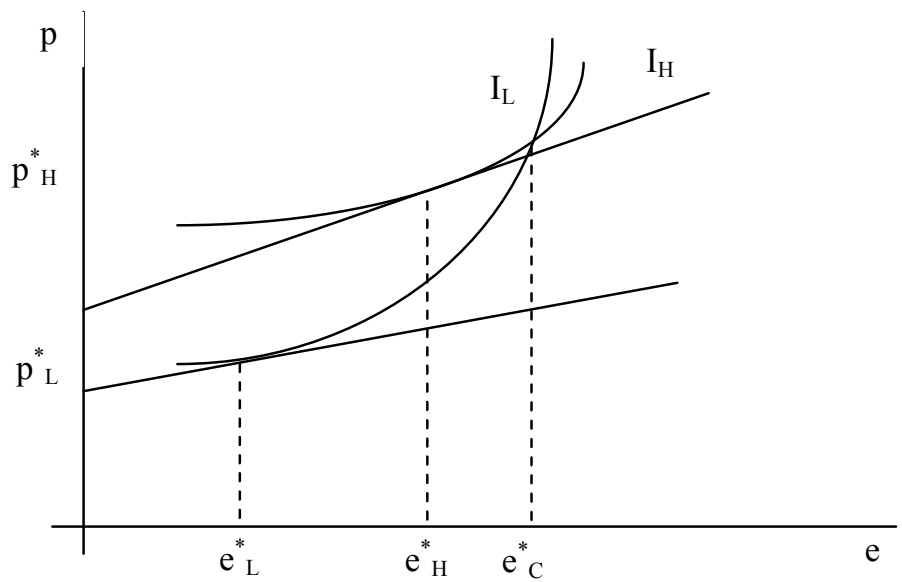

Fig. 3 The indifference curves with LTF envying HTF

In Fig. 3, the intersection point $e_{C}^{*}$ of the two types firms' indifference curves is right of $e_{H}^{*}$. If LTF fakes HTF, its indifference curve will move up and its utility increases. So LTF has the motivation to fake HTF. The case is more common in practice, and the equilibrium is more complexible. In general, it has three types of equilibrium solutions: pooling, separating and hybrid equilibriums. Belief held by game player should be employed. Each equilibrium has many specific solutions. According to some typical solutions, we do analysis with indifference curves. 
Pooling Equilibrium. Two types of firms can be pooled at a certain point. The belief held by consumers is $\mu(H \mid e)=\left\{\begin{array}{l}q, e=e_{p} \\ 0, e \neq e_{p}\end{array}\right.$. It means when emission reduction is $e_{p}$, consumers infer the probability of firm being $\mathrm{HTF}$ is $\mu\left(H \mid e_{p}\right)$, when emission reduction is not $e_{p}$, consumers confirm the firm is LTF. The strategy of consumers is $p(e)=\left\{\begin{array}{l}p_{p}, e=e_{p} \\ f(L, e), e \neq e_{p}\end{array}\right.$. In the expression, $p_{p}=q f\left(H, e_{p}\right)+(1-q) f\left(L, e_{p}\right)$. The strategy of firm is to select pooling point $e_{p}$ for every firm, which is expressed as $e(L)=e(H)=e_{p}$.

To make consumers' belief and strategy and firm's strategy to be pooling equilibrium, we resort to indifference curve figure again. The indifference curve of the strategy is as follows:

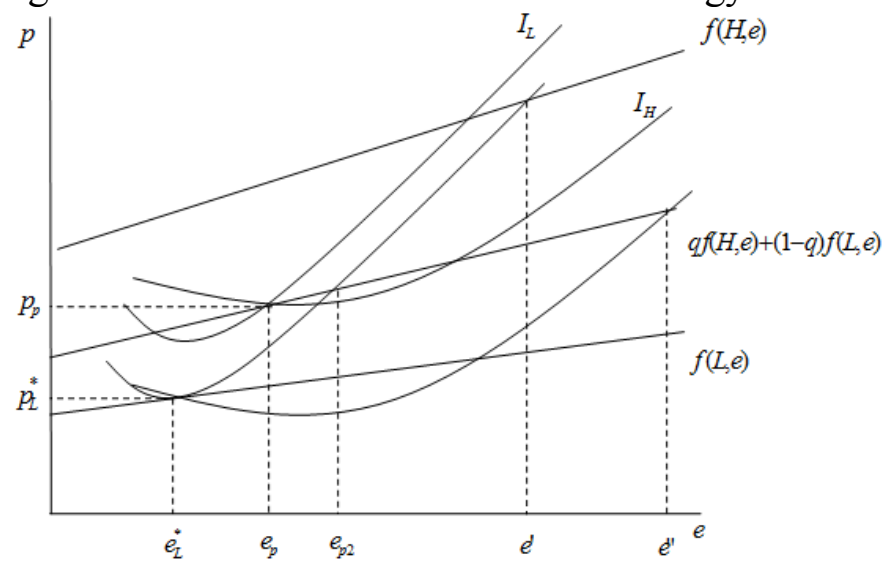

Fig. 4. The indifference curves of pooling equilibrium

There are some important parameters in Fig. 4. Value $e_{p}$ is the intersection of LTF indifference curve $I_{L}, \mathrm{HTF}$ indifference curve $I_{H}$ and curve $q f\left(H, e_{p}\right)+(1-q) f\left(L, e_{p}\right)$. Value $e_{p 2}$ is the intersection of $I_{L}$ with point $\left(e_{L}^{*}, p_{L}^{*}\right)$ and curve $q f\left(H, e_{p}\right)+(1-q) f\left(L, e_{p}\right)$. Value $e^{\prime}$ is the intersection of $I_{H}$ with point $\left(e_{L}^{*}, p_{L}^{*}\right)$ and curve $f(H, e)$. Value $e^{\prime \prime}$ is the intersection of $I_{H}$ with point $\left(e_{L}^{*}, p_{L}^{*}\right)$ and curve $q f\left(H, e_{p}\right)+(1-q) f\left(L, e_{p}\right)$. According to the property and position of the indifference curve, the strategy above is pooling equilibrium on the condition of $e_{p} \in\left[e_{L}^{*}, e_{p 2}\right]$. The pooling values, e.g. $e_{p}$, cannot be an interval. If $e_{p}$ is an interval, each of the two types of firms will select the emission at the intersection point of its indifference curve and $q f\left(H, e_{p}\right)+(1-q) f\left(L, e_{p}\right)$ respectively. But LTF will select the low value of the interval, and the HTF will select the high value of the interval. So it will not be pooling equilibrium but separating equilibrium. The interval of $e_{p} \in\left[e_{p 2}, e^{\prime \prime}\right]$ is separating district according to the described above, in which LTF will tell the truth to select $e_{L}^{*}$, but HTF will select the intersection of $I_{H}$ and $p_{p}$. This cannot be pooling equilibrium. The emission reduction is too high in $e_{p} \geq e^{\prime \prime}$, which leads to the case of HTF faking LTF. Each type of firms selects $e_{L}^{*}$ to be pooling equilibrium. But the pooling point is $\left(e_{L}^{*}, p_{L}^{*}\right)$.

At the same time, the emission reduction $e_{p}$ of the pooling point is positive correlation with q. In Fig. $4, e_{p 2}$ is the maximal value of pooling equilibrium. The extreme case is $\mathrm{q}=1$. This means consumers ensure the firm is HTF. So the condition $e_{p} \in\left[e_{H}^{*}, e^{\prime}\right]$ holds. The minimal value is $e_{H}^{*}$, which is the optimal value of HTF when it decides independently. The maximal value is $e^{\prime}$. The value of $\mathrm{q}$ is small in the market lack of credibility in China at present. The emission reduction at pooling point is small, too. So, building a good environment of credibility and integrity is helpful to the emission reduction of the pooling equilibrium. 
Furthermore in pooling equilibrium, the strategy of consumers at $e \neq e_{p}$ is a 'threat' strategy in fact. When firms do not select emission reduction $e_{p}$, consumers will take the price of $f(L, e)$ with punishment property whether HTF or LTF. As for pooling equilibrium, this strategy has no chance to be implemented. The function of it is to 'lock' firms adopting pooling reduction $e_{p}$ by 'eliminating' the possibility of firms selecting other reduction. But this threat is untrusted for the belief held by consumers has contradiction logically. Consumers think firm being LTF when the reduction is larger than $e_{p}$ but being HTF when the reduction is $e_{p}$. This is unreasonable. Denote the intersection of $I_{L}$ with pooling point and $f(H, e) e_{L}$, and the intersection of $I_{H}$ with pooling point and $f(H, e) e_{H}$. Then it is weak signal of LTF for $e \in\left(e_{L}, e_{H}\right)$. But the utility of HTF will improved if HTF let consumers believe it is HTF and give price $f(H, e)$. This will be contradict with pooling equilibrium.

So the pooling equilibrium is fragile only depending on the game players. It should resort to external forces, such as government and industrial association, to do mechanism design. By external behaviors, such as administrative regulation and industry self-discipline and etc., consumers' 'threat' strategy will have high credibility to improve the equilibrium self-enforced. Meanwhile, the external behaviors will have large effect on the construction of social credit environment.

Hybrid Equilibrium. In hybrid equilibrium, one firm selects a certain reduction, the other firm fakes by a probability. It has two cases. One is HTF selects a certain reduction, LTF pools HTF randomly, the other is reverse. The former is in common sense and more common. But the latter will appear when reduction is too large. For the analysis logic is consistent under two cases, the following analysis is based on the former.

In hybrid equilibrium, the consumer belief is post-probability. Prior probability is the probability of HTF endowed by Nature, which is denoted as q, too. As post-probability, consumer belief is modified according to Bayes rules.

Similar to pooling equilibrium, hybrid equilibrium does not have hybrid district. But in order to make strategy more completely, we will extend scopes covered by strategy. Denote the hybrid point $e_{H}$. The strategy of firm is that HTF select $e_{H}$, LTF select $e_{H}$ or $e_{L}^{*}$. The probability of LTF selecting $e_{H}$ is $\pi$.

The belief of consumers is $\mu(H \mid e)=\left\{\begin{array}{l}\frac{q}{q+(1-q) \pi}, e \geq e_{h} \\ 0, e<e_{h}\end{array}\right.$, which means consumers confirm LTF when reduction is smaller than $e_{H}$, but have a probability to be HTF while reduction is large than $e_{H}$. The probability can be obtained according to Bayes rules.

Then, the strategy of consumers is $p(e)=\left\{\begin{array}{l}\mu(H \mid e) f(H, e)+[1-\mu(H \mid e)] f(L, e), e \geq e_{h} \\ f(L, e), e<e_{h}\end{array}\right.$. At the hybrid point, consumers pricing expressed by expectations is as follows:

$$
p_{h}=\mu\left(H \mid e_{h}\right) f\left(H, e_{h}\right)+\left[1-\mu\left(H \mid e_{h}\right)\right] f\left(L, e_{h}\right)
$$

The strategy of LTF is selecting $e_{H}$ or $e_{L}^{*}$ when $e \geq e_{h}$. This means it is indifference between them, so $f\left(L, e_{L}^{*}\right)-c\left(L, e_{L}^{*}\right)=p_{h}-c\left(L, e_{h}\right)$.

On the basis of Nash equilibrium, we can validate the strategies of both consumer and firms are best response strategies. So, it is hybrid equilibrium. But we still need to determine the values and relationships of related parameters when it is hybrid equilibrium. It resorts to indifference curve too. The figure is as follows: 


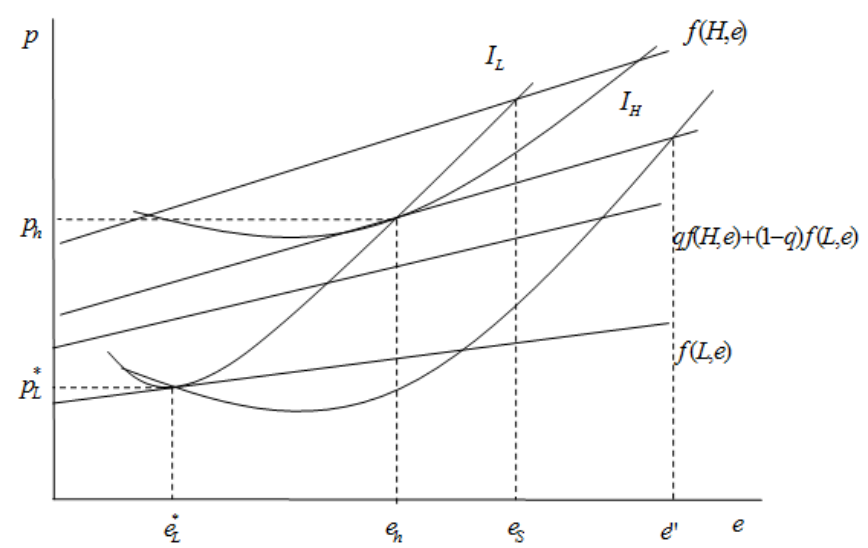

Fig. 5 The indifference curves of hybrid equilibrium

Due to $\mu(H \mid e)>q$, the strategy curve in hybrid equilibrium of consumer is high than the curve of prior probability, and the slope is steep also. Hybrid equilibrium will be separating equilibrium in the extreme case of $\mu(H \mid e)=1$ when the post-probability curve is coincident with curve $f(H, e)$. Hybrid equilibrium will become pooling equilibrium in the case of $\mu(H \mid e)=0$ when the post-probability curve is coincident with curve $f(L, e)$. The important parameters in Fig. 5 are as follows. Reduction $e_{h}$ at hybrid is the intersection of strategy curve of consumer with hybrid point , $I_{L}$ through point $\left(e_{L}^{*}, p_{L}^{*}\right)$ and $I_{H}$. Separating point $e_{S}$ is the intersection of $I_{L}$ with point $\left(e_{L}^{*}, p_{L}^{*}\right)$ and $f(H, e) . e^{n}$ is the intersection of $I_{H}$ with point $\left(e_{L}^{*}, p_{L}^{*}\right)$ and the strategy curve of with hybrid point. So compared with pooling equilibrium, hybrid equilibrium is the same in essence except for the probability value of consumers belief. From the analysis on pooling equilibrium, we can say with certainty that hybrid equilibrium is difficult to become a real equilibrium strategy from the rational perspective.

Separating Equilibrium. The no envy case described in Fig. 2 is the easiest separating refinement Bayes equilibrium to be implemented. In the equilibrium, firm's strategy is $\left(e(L)=e_{L}^{*}, e(L)=e_{L}^{*}\right)$, consumers' strategy is $\left(p\left(e_{L}^{*}\right)=p_{L}^{*}, p\left(e_{H}^{*}\right)=p_{H}^{*}\right)$. This means also that the belief of consumers is $\mu\left(H \mid e_{L}^{*}\right)=0, \mu\left(H \mid e_{H}^{*}\right)=1$. We should extend the strategy and belief of consumers to include deviating from the equilibrium path. So for the consumers, the strategy becomes $\mathrm{p}(e)=\left\{\begin{array}{l}p_{L}^{*}, e<e_{H}^{*} \\ p_{H}^{*}, e \geq e_{H}^{*}\end{array}\right.$, and the belief becomes $\mu(H \mid e)=\left\{\begin{array}{l}0, e<e_{H}^{*} \\ 1, e \geq e_{H}^{*}\end{array}\right.$.

Together with firm's strategy in the no envy case, it forms the expanding separating equilibrium. This equilibrium strategy has strong self-enforced. In fact, it is the refinement Bayes equilibrium.

Compared to Fig. 3 in the envy case, HTF need select a bigger reduction to achieve separating because LTF has the motivation to fake HTF. Denote the reduction $e_{S}$. We can form a separating equilibrium in envy case. In the equilibrium, the strategy of firm is $\left(e(L)=e_{L}^{*}, e(L)=e_{L}^{*}\right)$, the strategy of consumers is $\mathrm{p}(e)=\left\{\begin{array}{l}p_{L}^{*}, e<e_{S} \\ p_{H}^{*}, e \geq e_{S}\end{array}\right.$, the belief of consumers is $\mu(H \mid e)=\left\{\begin{array}{l}0, e<e_{S} \\ 1, e \geq e_{S}\end{array}\right.$. Then the indifference curve is as follows. 


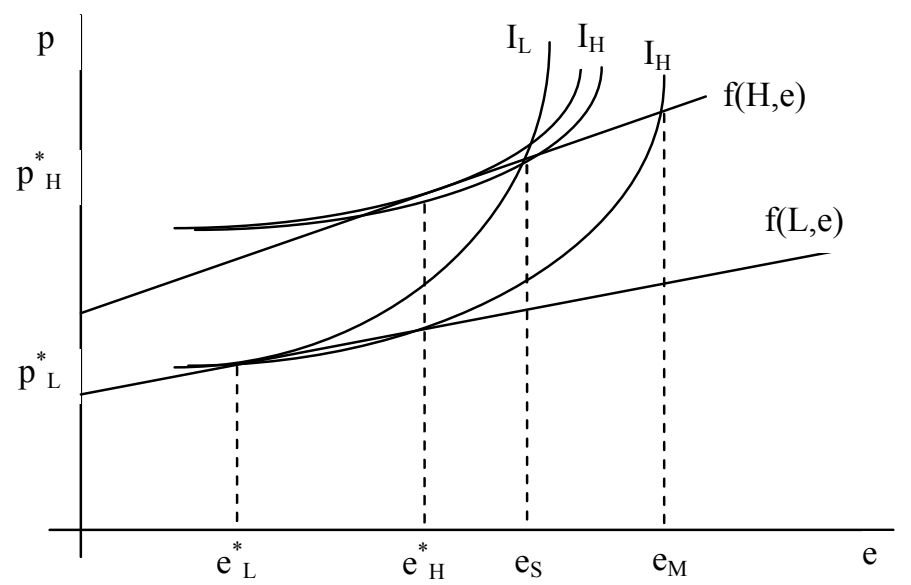

Fig. 6 The indifference curves of separating equilibrium

In Fig. 6, $\left(e_{S}, p_{S}\right)$ is the intersection of $I_{L}$ with $\left(e_{L}^{*}, p_{L}^{*}\right)$ and curve $f(H, e)$. Compared with the curve $I_{H}$ with $\left(e_{H}^{*}, p_{H}^{*}\right)$, the indifference curve of HTF moves down. So in this separating equilibrium, the utility of HTF will decline from its optimal state. It is the necessary costs due to envy or the cost structures of the two types of firms.

The point $\left(e_{L}^{*}, p_{L}^{*}\right)$ is not different with point $\left(e_{S}, p_{S}\right)$ for LTF. LTF will select reduction $e_{L}^{*}$ and $e_{S}$ randomly. So, the equilibrium is also the hybrid equilibrium described above, at which HTF will select fixed reduction and LTF will select from two point randomly. In fact, this point is the extreme point of hybrid equilibrium. If we suppose LTF always select $e_{L}^{*}$ at this case or more strictly, the reduction at separating point is little larger than $e_{S}$, then the strategy can be strictly separating equilibrium.

Of course, there are many separating equilibriums. As Fig. 6 shows, $e_{M}$ is the intersection of $I_{H}$ with point $\left(e_{L}^{*}, p_{L}^{*}\right)$ and curve $f(H, e)$. It can be validated easily that the separating equilibrium will be true for every $e$ when $e \in\left(e_{S}, e_{M}\right)$. But this is for HTF, it is no difference between $e_{M}$ and $e_{L}^{*}$, which is similar to the case of LTF. When $e>e_{M}$, the indifference curve of HTF will move down and the utility of HTF will decline. This will lead to pooling equilibrium for HTF has motivation to fake LTF. But for LTF, its utility does not change for its optimal selection is always $\left(e_{L}^{*}, p_{L}^{*}\right)$. So the point $\left(e_{S}, p_{S}\right)$ will be strict separating equilibrium. Compared with pooling equilibrium and hybrid equilibrium, separating equilibrium has strong self-enforced.

\section{Conclusions}

The paper takes product carbon footprint for firm as the signal to transfer its tech level and the quality of its product. Based the new perspective, it proposed a signal game model about pricing, emission reduction of firm and consumers. Then it analyzes the typical cases of pooling, hybrid and separating equilibriums of the model by indifference curve in qualitatively, and analyzes the feature and property of each equilibrium. It also compares the reduction setting, meaning and the relationships the three equilibriums. The results show that separating equilibrium is the only refinement Bayes Nash equilibrium and the self-enforced is strongest among them. In no envy case, separating equilibrium is the only Nash equilibrium, and is also the simplest and easiest in implementation. In envy case, separating equilibrium can make HTF select more reductions than in no envy case. For this paper focuses on qualitative analysis with indifference curve as tool, the results is directional and qualitative too. In order to get stronger conclusion, it need do further analysis by using explicit function. If there is supported by data and econometrics, the reliability and adaptability of research will be improved further. 


\section{Acknowledgements}

This work was financially supported by Beijing Key Laboratory (BZ0211), Beijing Intelligent Logistics System Collaborative Innovation Center.

\section{References}

[1] Christopher LW, Scott M H. Quantifying the global and distributional aspects of American household carbon footprint[J]. Ecological Economics, 2008, (66) : $379-391$.

[2] Paul P J, Anne C S, James H C, et al. A comparison of carbon calculators[J]. Environmental Impact Assessment Review, 2008,(28) : 106 - 115.

[3] Cholette S, Venkat K. The energy and carbon intensity of wine distribution: A study of logistical options for delivering wine to consumers[J]. Journal of Cleaner Production, 2009, 17(16):1401-1413.

[4] Absi N, Dauzère-Pérès S, Kedad-Sidhoum S, et al. The single-item green lot-sizing problem with fixed carbon emissions[J]. European Journal of Operational Research, 2016, 248(3):849-855.

[5] Hua G, Cheng T C E, Wang S. Managing carbon footprints in inventory management[J]. International Journal of Production Economics, 2011, 132(2):178-185.

[6] Chen X, Benjaafar S, Elomri A. The carbon-constrained EOQ[J]. Operations Research Letters, 2013, 41(2):172-179.

[7] Rui Z, Neighbour G, Han J, et al. Using game theory to describe strategy selection for environmental risk and carbon emissions reduction in the green supply chain[J]. Journal of Loss Prevention in the Process Industries, 2012, 25(6):927-936.

[8] Li S, Gu M. The effect of emission permit trading with banking on firm's production-inventory strategies[J]. International Journal of Production Economics, 2012, 137(2):304-308.

[9] Bai Q, Chen M. The distributionally robust newsvendor problem with dual sourcing under carbon tax and cap-and-trade regulations[J]. Computers \& Industrial Engineering, 2016, 98(C):260-274.

[10] Wakeland W, Sears L, Venkat K. Measuring the effects of food carbon footprint training on consumers: knowledge, attitudes, and behavioral intentions.[J]. Nuclear Instruments \& Methods, 2009, 21:444.

[11] Blengini G A, Shields D J. Green labels and sustainability reporting[J]. Management of Environmental Quality, 2010, 21(4):477-493.

[12] Shaofu Du, Lili Zhu, Liang Liang,et al. Emission-dependent supply chain and environment-policy-making in the 'cap-and-trade' system[J]. Energy Policy, 2013, 57(C):61-67.

[13] Hovelaque V, Bironneau L. The carbon-constrained EOQ model with carbon emission dependent demand[J]. International Journal of Production Economics, 2015, 164:285-291.

[14] Liu T, Wang Q, Su B. A review of carbon labeling: Standards, implementation, and impact[J]. Renewable \& Sustainable Energy Reviews, 2016, 53:68-79.

[15] Robert Gibbons. A Primer in Game Theory[M]. Beijing: China Social Sciences Press. 2015. 